\title{
Numerical Simulation of the Dispersion and Deposition of a Spray Carried by a Pulsating Airflow in a Patient-Specific Human Nasal Cavity
}

\author{
Ali Farnoud ${ }^{1 *}$, Xinguang $\mathrm{Cui}^{2}$, Ingo Baumann ${ }^{3}$, Eva Gutheil ${ }^{1}$ \\ ${ }^{1}$ Interdisciplinary Center for Scientific Computing, Heidelberg University, Germany \\ ${ }^{2}$ Lawrence Berkeley National Laboratory, Berkeley, CA \\ ${ }^{3}$ Department of Otorhinolaryngology, Head and Neck Surgery, Medical Center of Heidelberg \\ University, Heidelberg, Germany \\ *Corresponding author: ali.farnoud@iwr.uni-heidelberg.de
}

\begin{abstract}
The present numerical study concerns the dispersion and deposition of a nasal spray in a patient-specific human nose. The realistic three-dimensional geometry of the nasal cavity is reconstructed from computer tomography (CT) scans. Identification of the region of interest, removal of artifacts, segmentation, generation of the .STL file and the triangulated surface grid are performed using the software packages ImageJ, meshLab, and NeuRA2. An unstructured computational volume grid with approximately 15 million tetrahedral grid cells is generated using the software Ansys ICEM-CFD 11.0. An unsteady Eulerian-Lagrangian formulation is used to describe the airflow and the spray dispersion and deposition in the realistic human nasal airway using two-way coupling. A new solver called pimpleParcelFoam is developed, which combines the lagrangianParcel libraries with the pimpleFoam solver within the software package OpenFOAM 3.0.0. A large eddy simulation (LES) with the dynamic sub-grid scale (SGS) model is performed to study the spray in both a steady and a pulsating airflow with an inflow rate of $7.5 \mathrm{~L} / \mathrm{min}$ (or maximum value in case of the pulsating spray) and a frequency of $45 \mathrm{~Hz}$ for pulsation as used in commercial inhalation devices. 10,000 mono-disperse particles with the diameters of $2.4 \mu \mathrm{m}$ and $10 \mu \mathrm{m}$ are uniformly injected at the nostrils. In order to fulfil the stability conditions for the numerical solution, a constant time-step of $10^{-5} \mathrm{~s}$ is implemented. The simulations are performed for a real process time of $1 \mathrm{~s}$, since after the first second of the process, all particles have escaped through the pharynx or they are deposited at the surface of the nasal cavity. The numerical computations are performed on the high-performance computer bwForCluster MLS\&WISO Production using 256 processors, which take around 32 and 75 hours for steady and pulsating flow simulation, respectively. The study shows that the airflow velocity reaches its maximum values in the nasal valve, in parts of the septum and in the nasopharynx. A complex airflow is observed in the vestibule and in the nasopharynx region, which may directly affect the dispersion and deposition pattern of the spray. The results reveal that the spray tends to deposit in the nasal valve, the septum and in the nasopharynx due to the change in the direction of the airflow in these regions. Moreover, it is found that due to the pulsating airflow, the aerosols are more dispersed and penetrate deeper into the posterior regions and the meatuses where the connections to the sinuses reside.
\end{abstract}

\section{Keywords}

Particle deposition, nasal cavity, pulsating airflow, nebulized aerosol.

\section{Introduction}

The human nasal cavity is part of the upper respiratory system and it is connected to the face by the nostrils, which are the only visible parts of the nasal airway [1]. The nasal cavity is divided into right and left parts by the nasal septum and it is lined by a densely vascularized mucus [2]. The nasal passage consists of different regions which are illustrated in Figure 1: the nasal valve, inferior, middle and superior meatuses, the olfactory region and the nasopharynx. These complex regions inside the nasal cavity are related to the physiological functions of the nasal airway. For instance, the olfactory region which is lined by olfactory nerves, is responsible for the sense of smell. The complex system of the nasal cavity is responsible for filtering the inhaled particles available in air and cleaning the mucus layer by the mucociliary clearance process [1]. During inhalation, the airflow and the particles enter the nasal cavity at the nostrils, pass through a 90 degree bend

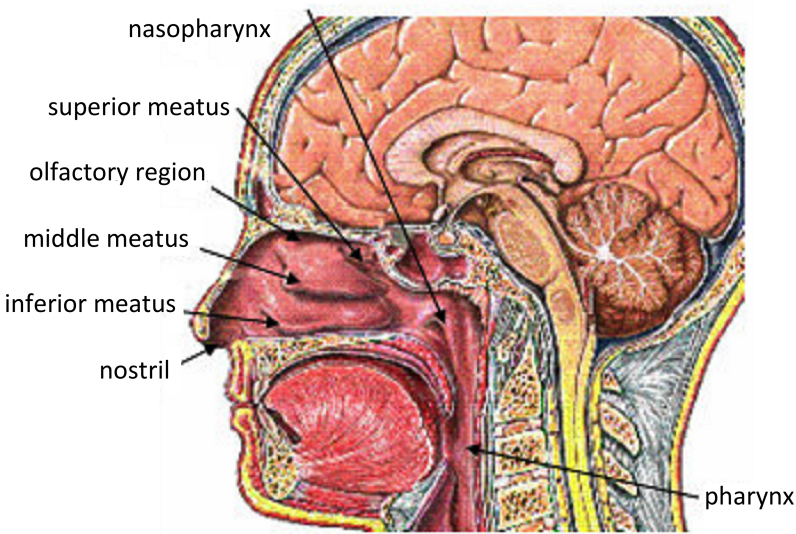

Figure 1. The anatomy of the nasal cavity [4] and a constricted area (nasal valve). Afterwards, the air and the particles enter the main nasal cavity, where they pass through the three airways of inferior, middle, and superior meatuses. The streaming air from the meatuses 
finally unifies in the nasopharynx and experiences another 90 degree bend which connects the nasal cavity with the pharynx [1]. The complex geometry of the meatuses and two 90 degree bends at the nasal valve and the nasopharynx help the respiratory system to trap the bacteria and potentially toxic particles on the sticky mucus. The particles that are trapped on the mucus layer finally dissolve in the mucus layer and absorb into the blood [2].

The nasal cavity is surrounded by paranasal sinuses which are air-filled cavities. There are four pairs of the sinuses which are connected to the main nasal airway: the maxillary, the ethmoid, the frontal and the sphenoid sinuses. The paranasal sinuses have various functions such as reducing the weight of the skull and resonating the speech [1]. These normal functions of the nose may be disturbed by various serious health conditions.

Chronic rhinosinusitis (CRS) is a common upper respiratory disease, which is defined as the inflammation of the nasal and paranasal cavity for the duration of more than 12 weeks [5]. CRS could occur due to the bacterial, fungal or viral infections, allergies, exposure to the inhaled irritants, septum deviation, or nasal polyps. The symptoms of CRS are mucosal swelling, mucus secretion into the nasopharynx, loss of cilia, airway obstruction, and no sinus drainage [1]. The prevalence of CRS disorder in Europe and USA is $10.9 \%$ [6] and 9-14\%, respectively [7]. The quality of life (QOL) of CRS patients is significantly effected by this disorder. Functional Endoscopic Sinus Surgery (FESS) is one of the main solutions for the patients suffering from CRS which has a success rate of 70-90\% [8,9]. It is found that after FESS there is a remarkable improvement in the QOL of the CRS patients [10].

The nasal airway is a potential path for drug delivery for CRS patients due its large surface area and its vascular surface [3]. However, conventional nasal drug delivery devices could not efficiently transport the drug to the desired target. Since CRS occurs in sinuses and connections of the sinuses which are not ventilated properly, the use of a conventional nasal sprays leads to an inefficient delivery of the medication. By using a nasal pump sprays, the particles tend to deposit in the anterior parts of the nasal airway [11] and medication cannot penetrate into the posterior regions of the nasal cavity, the ostia and the sinuses. This is due to the high inertial impaction caused by large particles with diameters of around $60 \mu \mathrm{m}$ [11]. Recent nebulizers are producing smaller particles in the range of $1-10 \mu \mathrm{m}$ [11], which could lead to an even distribution of the particles and and improvement of the penetration of the particles to posterior parts of the nasal cavity. Accordingly, it is important to study the airflow pattern and the particle dispersion and deposition in different patient-specific nasal cavities. Generating a pulsating airflow may improve the penetration of the airflow into the meatuses which are the connections to the sinuses [11]. Conclusively, the accessibility of the medication into the meatuses and sinuses is improved. Möller et al. [11] experimentally showed that the implementation of a pulsating airflow improves the delivery of the aerosols into the posterior regions of the nasal cavity and the sinuses. They experimentally compared a nasal pump spray and a pulsating aerosol delivery device. They concluded that the utilization of the nasal pump spray led to deposition of the most of the aerosols in the nasal valve region. Hilton et al. [12] experimentally studied the effect of the diameter of the particles on the deposition in the paranasal sinuses. They found that the particles with a diameter smaller than $5 \mu \mathrm{m}$ led to a higher deposition efficiency compared to the particles with diameters larger than $5 \mu \mathrm{m}$.

In order to gain a better understanding on the parameters affecting the airflow pattern and the aerosol dispersion and deposition in the nasal cavity, several in-vivo $[13,14,15]$ and in-vitro $[16,17,18]$ studies have been performed. Since performing experimental measurements in several human nasal airway models are time consuming, and technical difficulties with respect to the precision of in-vivo measurement due to complex geometry of the nose as well as unwanted side effects caused by the probes exist that may affect the gas flow [19], computational fluid dynamic (CFD) studies of the nasal airflow are an affordable alternative. In addition, CFD allows for parametric studies of variables which are difficult to change in the experiment. The advancement of CFD as well as the digital image processing sciences in the recent decades allows for a precise simulation of the nasal airflow and the particle dispersion and deposition pattern in the patient-specific nasal cavity. The first numerical studies of the airflow pattern in the nasal airway were accomplished by Keyhani et al. [20] and Subramaniam et al. [21]. Recently, particle dispersion and deposition have been studied in realistic nasal airways [22, 23, 24]. These simulations were performed with the commercial software packages CFX [22] and Fluent [23, 24]. Most of the research in this area considers a steady inlet airflow [25] or an unsteady flow representing inhalation-exhalation cycles [26]. Frank-Ito et al. [27] studied the effect of the volume grid cells on the deposition efficiency of the particles while modeling the realistic airflow and particle transport in a realistic human cavity including the paranasal sinuses. They found that by increasing the number of the tetrahedral elements and specially the density of the volume grid cells at the wall, the particle deposition fraction decreased. They concluded that a coarse mesh could lead to overestimation of the deposition efficiency of the particles.

In the present study, a patient-specific geometry of the nasal airway (without sinuses) is constructed from CT images, and the effect of the pulsating airflow on the dispersion and deposition of the particles in the nasal airway is studied using a large eddy simulation. Moreover, the effect of the diameter of the particles on the deposition efficiency in the nasal cavity is investigated.

\section{Methods}

The airflow and particle dispersion and deposition during steady and pulsating inlet airflow conditions is studied in a CFD simulation. The steps involved are the identification of the region of interest in the CT scan and the generation of a numerical surface and volume grids for the simulation. After determination of appropriate governing equations, a large eddy simulation (LES) is performed with appropriate boundary and inlet conditions. 


\section{Geometry}

A realistic three-dimensional geometry of the nasal cavity is constructed from the CT images of a patient [28]. The images with slice thickness of $1 \mathrm{~mm}$ are obtained in .DICOM format. Subsequently, identification of the region of interest, artifacts removal, segmentation, extraction of the .STL file and generation of the triangulated surface are preformed by using the software packages ImageJ, meshLab, NeuRA2 [28]. Due to complexity of the geometry including sharp curves and edges, a volume grid with approximately 15 million unstructured tetrahedral cells is generated using the software package ICEM-CFD Ansys 11.0. Figure 2 illustrates the sagittal view of the CT images (left) and the constructed geometry (right). The airflow enters from the inlet and leaves the nasal passage from the outlet.
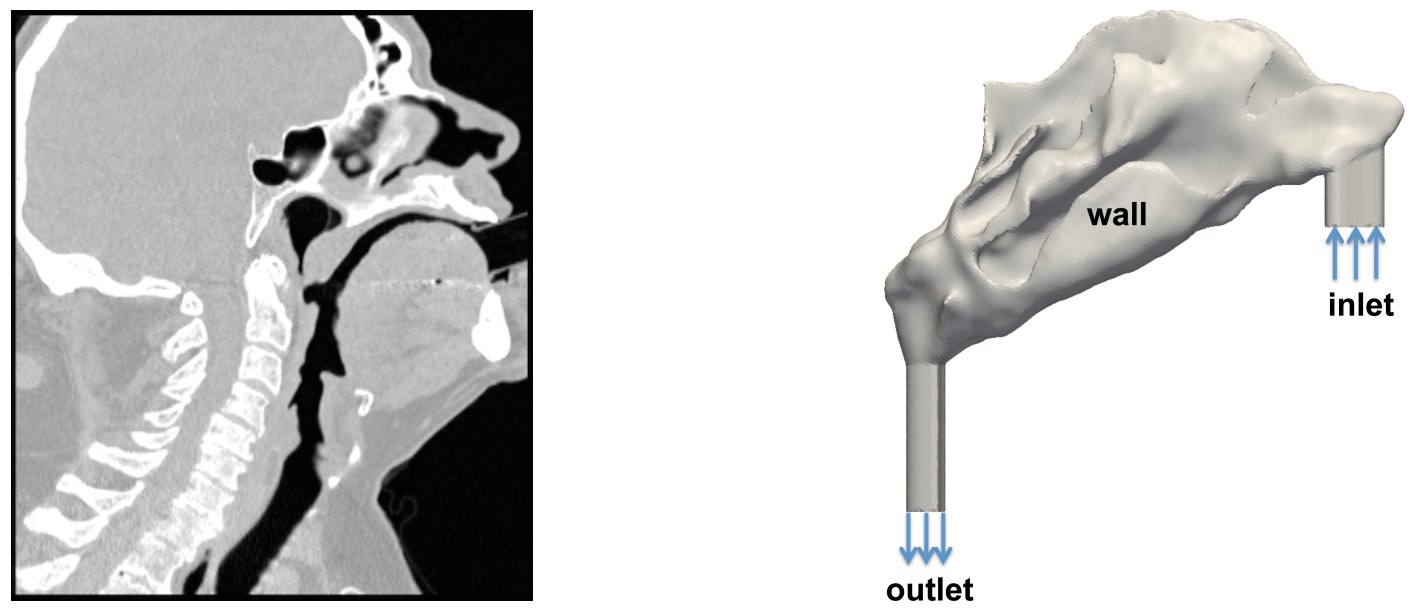

Figure 2. The CT image of the patient (left) and the constructed three dimensional nasal cavity (right)

\section{Governing Equations}

A Eulerian-Lagrangian description of the gas and liquid phases is employed where a large eddy simulation with the dynamic sub-grid scale model [32] is used to describe the laminar-transitional-turbulent gas flow. Two-way coupling [28] of the gas-phase equations with the particles is used.

In the Smagorinsky SGS model, the grid filter $\bar{G}$

$$
\bar{f}(\mathbf{x})=\int_{D} f\left(\mathbf{x}^{\prime}\right) \bar{G}\left(\mathbf{x}, \mathbf{x}^{\prime}\right) \mathrm{d} \mathbf{x}^{\prime}
$$

with grid-dependent filter width $\bar{\Delta}$ is applied to both the continuity and the momentum equations. The sub-grid stress tensor $\tau_{i j}^{\mathrm{r}}$ appearing in the filtered equations [31] is closed using an eddy viscosity hypothesis $\tau_{i j}^{\mathrm{r}}-\left(\delta_{i j} / 3\right) \tau_{k k}^{\mathrm{r}} \approx$ $-2 v_{\mathrm{r}} \bar{S}_{i j}$, where the residual stress is related to the filtered rate of strain, $\bar{S}_{i j}$. The eddy viscosity then is closed using the Smagorinsky coefficient $C_{\mathrm{S}}, v_{\mathrm{r}}=\left(C_{\mathrm{S}} \bar{\Delta}\right)^{2}|\bar{S}|$ with $\bar{S}_{i j}=1 / 2\left(\partial \bar{U}_{i} / \partial x_{j}+\partial \bar{U}_{j} / \partial x_{i}\right)$ and $|\bar{S}|=\left(2 \bar{S}_{i j} \bar{S}_{i j}\right)^{1 / 2}$ [31] to yield

$$
\tau_{i j}^{\mathrm{r}}-\left(\delta_{i j} / 3\right) \tau_{k k}^{\mathrm{r}} \approx-2 C_{\mathrm{s}} \bar{\Delta}^{2}|\bar{S}| \bar{S}_{i j} .
$$

The assumption of a constant Smagorinsky coefficient is relaxed in the dynamic SGS model [29], where a so-called test filter $\widetilde{G}$ with filter width $\widetilde{\Delta}$

$$
\widetilde{f}(\mathbf{x})=\int_{D} f\left(\mathbf{x}^{\prime}\right) \widetilde{G}\left(\mathbf{x}, \mathbf{x}^{\prime}\right) \mathrm{d} \mathbf{x}^{\prime}
$$

is performed after the filtering given by Eq. (1). Then the continuity and momentum equations for an unsteady, incompressible and isothermal airflow field yield [29]

$$
\begin{aligned}
& \frac{\partial \widetilde{\bar{U}}_{j}}{\partial x_{j}}=0 \\
& \frac{\partial \widetilde{\bar{U}}_{i}}{\partial t}=-\frac{\partial\left(\widetilde{\bar{U}}_{i} \widetilde{\bar{U}}_{j}\right)}{\partial x_{j}}-\frac{\partial \widetilde{\bar{p}}}{\partial x_{i}}-\frac{\partial T_{i j}}{\partial x_{j}}+\frac{1}{\operatorname{Re}} \frac{\partial^{2} \widetilde{\bar{U}}_{i}}{\partial x_{j} \partial x_{j}}+\widetilde{\bar{S}}_{\mathrm{p}, i}
\end{aligned}
$$

In the equations above, $i, j=1, \ldots, 3$, and the Einstein summation convention is used. Note that in the momentum equation (5), the filtered source term $\widetilde{\bar{S}}_{\mathrm{p}, i}$

$$
\widetilde{\bar{S}}_{\mathrm{p}, i}=\sum_{i=1}^{n}\left[\frac{m_{\mathrm{p}, i}}{\Delta V} \frac{\left(\mathbf{U}_{\mathrm{p}, i}\right)_{t_{\mathrm{out}}}-\left(\mathbf{U}_{\mathrm{p}, i}\right)_{t_{\mathrm{in}}}}{t_{\mathrm{out}}-t_{\mathrm{in}}}\right]
$$


appears that accounts for the interaction of the particles and gas phase, i.e. two-way coupling is used [28]. For one-way coupling, the effect of particle motion on the gas phase is neglected, i.e. the source term in Eq. (5), $S_{\mathrm{p}, i}$ is set to zero. $T_{i j}$ is the dynamic Smagorinsky stress tensor, which replaces the residual stress tensor $\tau_{i j}{ }^{r}$ used for the constant Smagorinsky-coefficient approach above. The sub-grid stress tensor then yields [29] $T_{i j}=\widetilde{U_{i} U_{j}}-\widetilde{\bar{U}}_{i} \widetilde{\bar{U}}_{j}$, which is related to the resolved turbulent stress $L_{i j}=\widetilde{\bar{U}}_{i} \bar{U}_{j}-\widetilde{\bar{U}}_{i} \widetilde{\bar{U}}_{j}$ through the Germano identity [29]

$$
L_{i j}=T_{i j}-\tilde{\tau}_{i j}^{\mathrm{r}}
$$

In analogy to the Smagorinsky stress tensor $\tau_{i j}^{\mathrm{r}}$, the dynamic Smagorinsky stress tensor is described through the viscosity hypothesis as [29]

$$
T_{i j}-\left(\delta_{i j} / 3\right) T_{k k} \approx-2 \widetilde{\bar{\Delta}}^{2} \mid \widetilde{S}^{\mid} \widetilde{\bar{S}}_{i j}
$$

After substitution of the test-filtered Eq. (2) and Eq. (8) in the Eq. (7) and multiplying both sides of the equation by $\bar{S}_{i j}$, and finally averaging both sides of the equations, the Smagorinsky constant $C_{\mathrm{s}}$ is derived as [29]

$$
C_{\mathrm{s}}=-\frac{1}{2} \frac{\left\langle L_{k l} \bar{S}_{k l}\right\rangle}{\widetilde{\bar{\Delta}}^{2}\left\langle\mid \tilde{\bar{S}}_{\bar{S}} \tilde{\bar{S}}_{m n} \bar{S}_{m n}\right\rangle-\bar{\Delta}^{2}\left\langle\widetilde{\tilde{S}}_{\mid \bar{S}} \bar{S}_{p q}\right\rangle}
$$

The Smagorinsky coefficient $C_{\mathrm{s}}$ is a function of time and space and is locally calculated in every time step, leading to a more appropriate calculation of $C_{\mathrm{s}}$ for laminar-transitional-turbulent flows [29].

The mono-disperse particles are uniformly injected through both nostrils, and the initial particle velocity are taken equal to the airflow velocity at the nostrils. Lagrangian particle tracking is used to calculate the location $\mathbf{x}_{\mathrm{p}}$ and the velocity $\mathbf{U}_{\mathrm{p}}$ of the particles

$$
\begin{aligned}
& \frac{\mathrm{d} \mathbf{x}_{\mathrm{p}}}{\mathrm{d} t}=\mathbf{U}_{\mathrm{p}} \\
& \frac{\mathrm{d} \mathbf{U}_{\mathrm{p}}}{\mathrm{d} t}=\mathbf{F}_{\mathrm{D}}+\mathbf{g}=\frac{18 \mu}{\rho_{\mathrm{p}} d_{\mathrm{p}}^{2}} \frac{C_{\mathrm{D}} \operatorname{Re}_{\mathrm{p}}}{24}\left(\widetilde{\widetilde{\mathbf{U}}}-\mathbf{U}_{\mathrm{p}}\right)+\mathbf{g},
\end{aligned}
$$

where drag $\mathbf{F}_{\mathrm{D}}$ and gravitational forces $\mathbf{g}$ are exerted on the particles, and the drag coefficient $C_{\mathrm{D}}$ is [30]

$$
C_{\mathrm{D}}= \begin{cases}\frac{24}{\operatorname{Re}_{\mathrm{p}}} & \text { if } \mathrm{Re}_{\mathrm{p}} \leq 10^{3} \\ \frac{24}{\operatorname{Re}_{\mathrm{p}}}\left(1+0.15 \mathrm{Re}_{\mathrm{p}}^{0.678}\right) & \text { if } \mathrm{Re}_{\mathrm{p}}>10^{3} .\end{cases}
$$

In Eq. (11), $\mu$ is the dynamic gas viscosity, $\operatorname{Re}_{\mathrm{p}}$ denotes the particle Reynolds number, and $\rho_{\mathrm{p}}$ is the particle density.

\section{Physical properties and boundary conditions}

The physical properties of air are considered for the gas phase, and for the particles, the properties of water are assigned since most of the drugs are aqueous solutions with the approximate density of water [32]. Due to the stickiness of the mucus layer on the inner surface of the nasal airway, it is assumed that the particles are trapped on the mucus once they hit the wall. The particles which are trapped on the surface of the mucus move towards the pharynx via the mucociliary clearance function of the nasal cavity. During the mucociliary clearance process, the movement of the cillia directs the particles towards the pharynx. However, since the velocity of the particles during the mucociliary clearance process is very slow in comparison to both the velocity of the air and the particle motion in the nasal cavity, it is reasonable to consider the nasal wall as stationary (no-slip) [33]. In the present study it is assumed that the wall is smooth and stationary. In order to control the inlet and outlet effects, the inlet and the outlet of the nasal cavity are

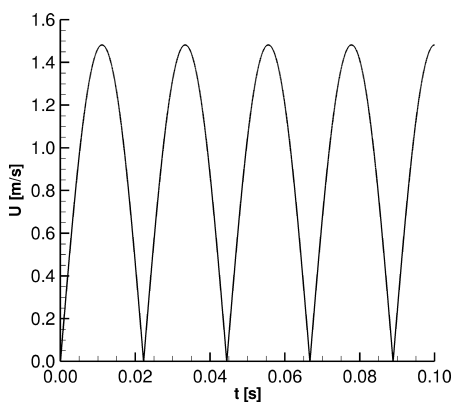

Figure 3. Profile of the pulsating air inflow extended by pipes [34] which are shown in the right part of Figure 2.

The present study compares steady and pulsating airflow at the inlet of the nostrils. For the steady inhalation, a constant airflow rate of $7.5 \mathrm{~L} / \mathrm{min}$ is considered. In case of the pulsating airflow, a sinusoidal shape with a frequency of $45 \mathrm{~Hz}$ and an maximum value of $7.5 \mathrm{~L} / \mathrm{min}$ is used, cf. Figure 3. This condition corresponds to $63.7 \%$ of the present steady inhalation rate. The pulsating flow is described by

$$
U=U_{0}\left|\sin \left(2 \pi \omega^{*} t\right)\right|
$$

with $\omega^{*}=\omega / 2$ and $\omega=45 \mathrm{~Hz}$. A total of 10,000 mono-disperse particles are injected at the nostrils with the corresponding initial gas velocity of $1.48 \mathrm{~m} / \mathrm{s}$ over the first time step of $10^{-5} \mathrm{~s}$ [35]. In order to study the effect of the particle diameter on the deposition efficiency of the particles in the nasal airway, two different particle diameters of $2.4 \mu \mathrm{m}$ and $10 \mu \mathrm{m}$ at fixed particle number density are studied. The particle diameter of $2.4 \mu \mathrm{m}$ is selected since it represents the Sauter mean diameter of the aerosols generated by the PARI SINUS nebulizers [11]. 


\section{Numerical method}

The governing equations of the gas flow and those for particle motion are solved by a hybrid Eulerian-Lagrangian solver. The LES simulations with the dynamic SGS model are performed using the solver pimpleFoam in the software package OpenFOAM 3.0.0, which includes the PIMPLE (PISO combined with SIMPLE) algorithm to couple the gas phase pressure and the gas velocity. This solver is coupled to the lagraniganParcel library, and a new solver called pimpleParcelFoam is developed, which enables the two-way coupled Eulerian-Lagrangian solution for the airflow and the particles. This numerical model is suitable to solve a three-dimensional, transient, incompressible, isothermal and Newtonian gas phase flow with particle motion. The simulations are carried out for a real process time of $1 \mathrm{~s}$, since after the first second more than $99 \%$ of the particle are attached to the surface of the wall or have escaped from the outlet. The computations are performed on the bwForCluster MLS\&WISO using 256 processors with a constant time step of $10^{-5} \mathrm{~s}$ for both the gas and particle phases. For the simulations with steady and pulsating airflow, the clock times are approximately 32 and 75 hours, respectively.

\section{Results and discussion}

First, the gas flow is analyzed for both the steady and pulsating flows before the particle dispersion and deposition is discussed for the different conditions under consideration.

\section{Gas phase}

Figure 4 shows the contour plot of the gas velocity at different coronal cross-sections with equal intervals of $9.22 \mathrm{~mm}$ between the nasal valve (right) and nasopharynx (right) for steady inhalation. The airflow velocity attains its maximum values in the nasal valve region, the nasopharynx and the inferior parts of the septum. The olfactory region and the tips of the meatuses are not ventilated in accordance with results by Chung and Kim [36]. Figure 5 illustrates the streamlines of the gas velocity for steady inhalation. The steady airflow uniformly enters the nostrils

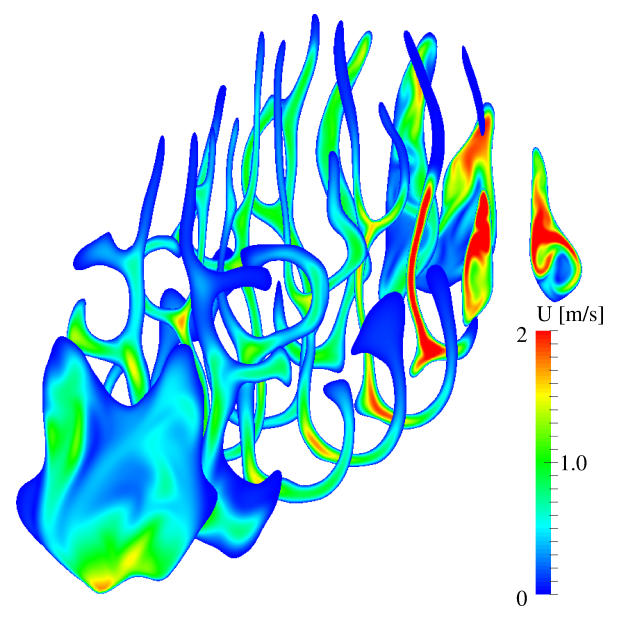

Figure 4. Gas velocity for steady inhalation at different coronal planes between the nasal valve on the right, and the nasopharynx on the left, cf. Figure 1, right part

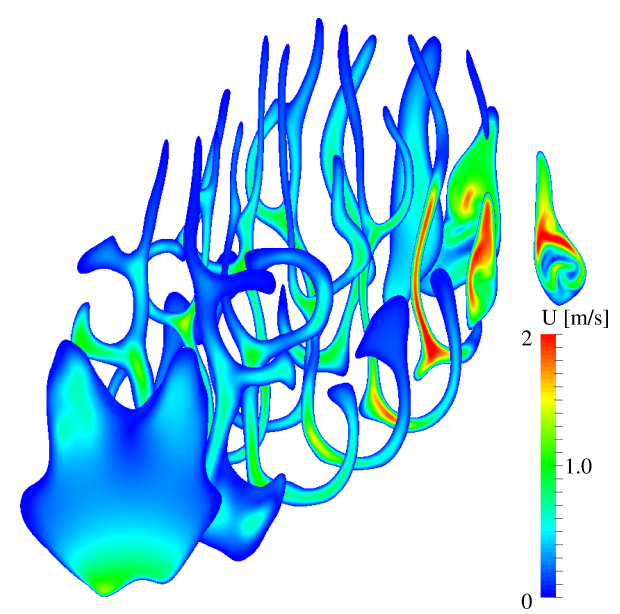

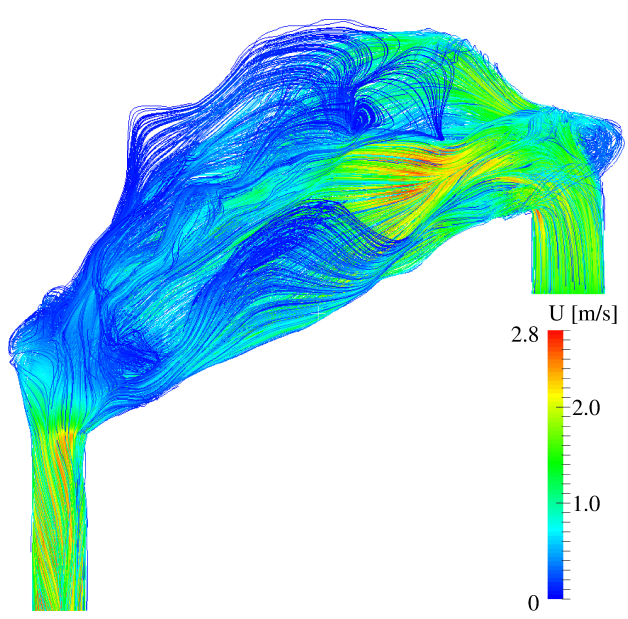

Figure 5. Streamlines of the gas velocity for steady inhalation

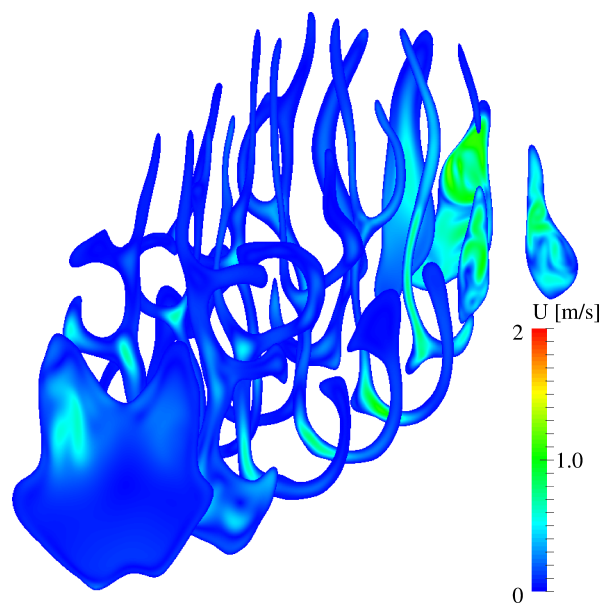

Figure 6. Pulsating airflow: gas velocity at different cross-sections for the second pulse left: at $(t=0.0333 \mathrm{~s})$ and right: at $(t=0.0444 \mathrm{~s})$, cf. Figure 3 
and passes through a $90^{\circ}$ bend at the vestibule, inducing vortices and a complex aflow pattern. Depending on the patient-specific anatomy of the nasal cavity, the location and shape of the vortices in the airway may change [21]. In the present study, the complex flow and vortices in the nasal valve region and immediately after the nasal valve are similar to experimental results of Doorly et al. [19]. The airflow field in the nasal cavity plays a quite important role, since the best treatment method for each patient may be decided based on the flow conditions in nasal passage [36]. The airflow from the upper, middle and lower meatuses combine and unify in the nasopharynx region. The combination of the flows from the three meatuses and a $90^{\circ}$ bend in the nasopharynx causes complex vortices and a swirling flow in the nasopharynx region.

Figure 6 shows the corresponding contour plots of the gas velocity for the pulsating flow during the second pulse, cf. Figure 3 , at the peak at $t=0.0333 \mathrm{~s}$ (left) and at the end of the second pulse at $t=0.0444 \mathrm{~s}$ (right). A comparison of the steady and pulsating inhalation shows not much difference at the peak of the second pulse except for that the absolute values are somewhat reduced for the pulsating flow. This is due to the fact that the steady inflow occurs at a constant flow rate of $7.5 \mathrm{~L} / \mathrm{min}$ whereas this value is only reached at the peak of the pulse during pulsating inflow, leading to an overall (integral) average value of $4.78 \mathrm{~L} / \mathrm{min}$. At the end of the second pulse, cf. Figure 6 , the velocity of the gas phase in the nasal airway is dramatically reduced except for in the upper parts of the nasal valve. A future simulation of an average pulsed air inflow rate of $7.5 \mathrm{~L} / \mathrm{min}$ may help to a better evaluation of the differences in results of steady versus pulsating airflow.

\section{Particle dispersion and deposition}

This section concerns the study of the influence of the steady and pulsating particle injection as well as the particle size on the particle dispersion and deposition efficiency. Two different particle diameters of $2.4 \mu \mathrm{m}$ and $10 \mu \mathrm{m}$ are studied for a fixed value of 10,000 mono-disperse particles that are injected uniformly at the nostrils.

Figure 7 depicts the evolution of the particles with a diameter of $2.4 \mu \mathrm{m}$, carried by a pulsating airflow at different times, cf. Figure 3. At $t=0.00555 \mathrm{~s}$, which is at half of the ascending part of the first pulse, the particles reside in the extended inlet pipe. At $t=0.0222 \mathrm{~s}$, i.e. at the end of the first pulse, the particles pass through the nasal valve and enter into the main nasal cavity. At the peak of the third pulse at $t=0.0555 \mathrm{~s}$, the particles enter the nasopharynx, the inferior and the middle meatuses. At the end of the fifteenth pulse at $t=0.333 \mathrm{~s}$, some particles have moved out of the domain towards the pharynx, some are attached to the wall, and others are still moving inside the nasal airway. In general, the particles tend to deposit in the nasal valve, nasopharynx and some parts of the septum. In particular, particles penetrate into the tips of the meatuses or into the upper and posterior parts of the nasal cavity. Figure 8 illustrates the corresponding particle evolution of the same particle size carried by the steady airflow. Even though the principal pattern of particle evolution and deposition is similar for steady airflow, the aerosols penetrate deeper into the posterior regions and the meatuses for the pulsating airflow, where the connections to the sinuses reside, see Figure 7.

It is observed that the particle evolution occurs faster for steady inhalation compared to the current pulsating flow condition which, however, corresponds to only $63.72 \%$ of the steady inhalation rate. A better evaluation of the different conditions with respect to the particle evolution rate may be achieved if the present maximum value of the
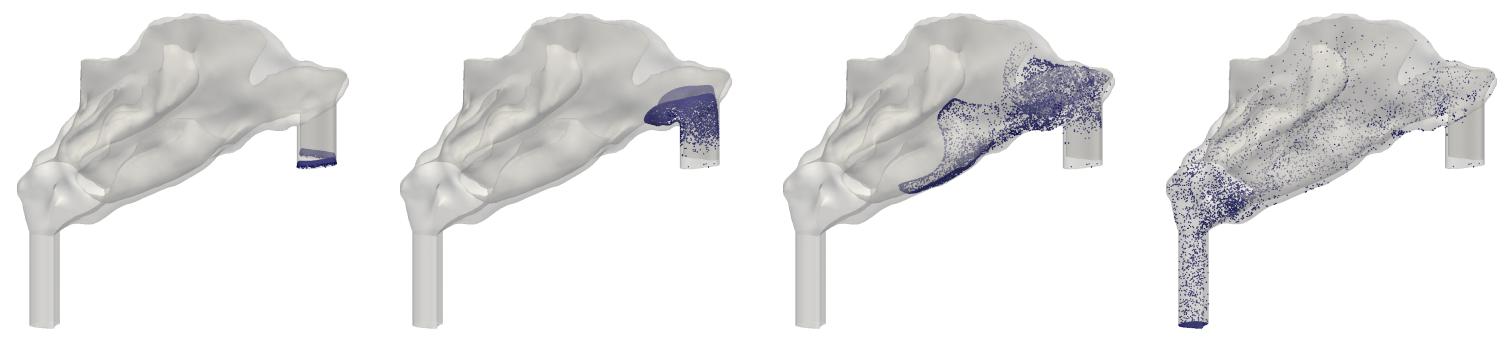

Figure 7. Temporal evolution of the particles of diameter $2.4 \mu \mathrm{m}$ for the pulsating airflow from left to right at $t=0.00555 \mathrm{~s}$, $t=0.0222 \mathrm{~s}, t=0.0555 \mathrm{~s}$, and $t=0.333 \mathrm{~s}$
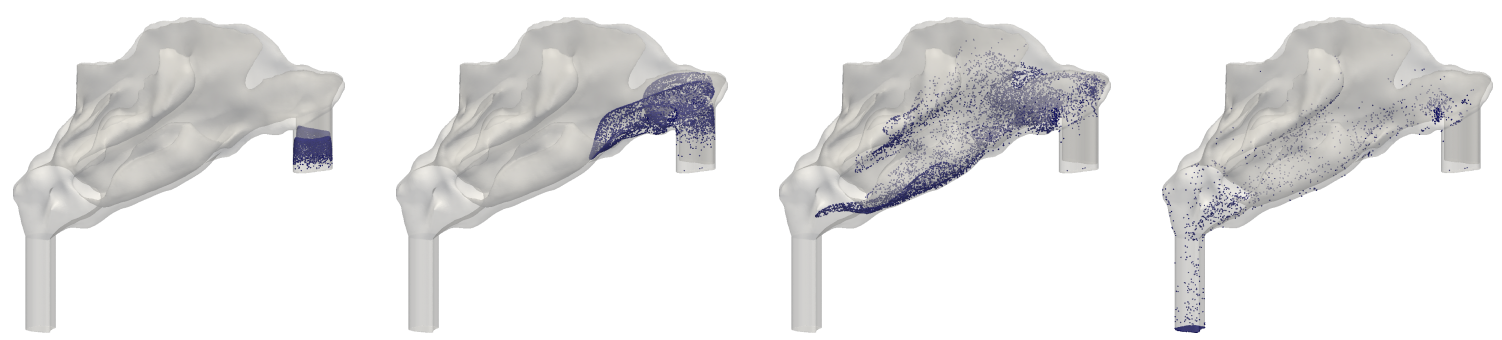

Figure 8. Temporal evolution of the particles of diameter $2.4 \mu \mathrm{m}$ for the steady inhalation from left to right at $t=0.00555 \mathrm{~s}$, $t=0.0222 \mathrm{~s}, t=0.0555 \mathrm{~s}$, and $t=0.333 \mathrm{~s}$ 


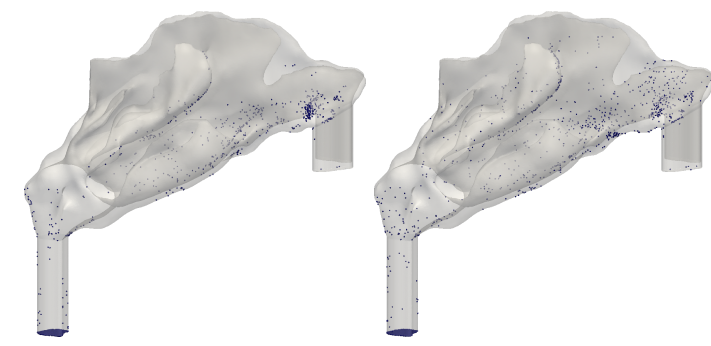

Figure 9. Particle deposition pattern, $d_{0}=2.4 \mu \mathrm{m}$ after $1 \mathrm{~s}$ for left: steady and right: pulsating airflow

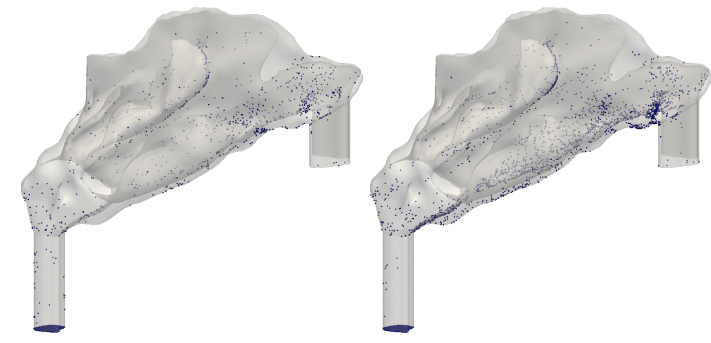

Figure 10. Particle deposition pattern, $d_{0}=2.4 \mu \mathrm{m}$ (left) and $d_{0}=10.0 \mu \mathrm{m}$ (right) after $1 \mathrm{~s}$ for the pulsating airflow

pulsating flow should be enhanced to 1.56 times of the initial velocity of the steady inhalation, resulting in the same integral inhalation rate for both situations.

Figure 9 illustrates the deposition pattern of the particles with diameter of $2.4 \mu \mathrm{m}$ after $1 \mathrm{~s}$ for steady (left) and pulsating (right) airflow. Steady airflow leads to accumulation of particle deposition in some regions of the nasal airway, in particular in the nasal valve and the nasal pharynx. The pulsating airflow leads to improved ventilation of the olfactory region, the superior meatuses and the tips of the middle meatuses, which is associated with particles deposition in theses regions. However, for the simulation of the steady airflow, no deposition is observed in the olfactory region, superior meatuses and tips of the middle meatuses.

The variation of initial particle diameter for steady inhalation shows that an increase of the particle diameter from $2.4 \mu \mathrm{m}$ to $10 \mu \mathrm{m}$ results in an enhancement of the deposition efficiency (DE) in the nasal cavity from $7.4 \%$ to $24.8 \%$ (not shown). The larger particles are trapped on the surface of the nasal cavity due to higher inertial impaction [11]. However, smaller particles penetrate deeper into the nasal airway and they enter the pharynx. For this reason, nasal drug delivery devices which are designed to target the drug into the lower respiratory system, are to generate finer particles. A similar behavior is observed for the pulsating airflow with particle diameters of $2.4 \mu \mathrm{m}$ and $10 \mu \mathrm{m}$ shown in Figure 10. A variation of the particle diameters of $2.4 \mu \mathrm{m}$ and $10 \mu \mathrm{m}$ results in deposition efficiencies of $8.3 \%$ and $24.8 \%$, respectively. The DE for the steady and pulsating flow carrying particles with a diameter of $10 \mu \mathrm{m}$ lead to the same results with a different deposition pattern showing that the pulsating spray shows less particle accumulation and is favored for the particles to reach into the meatuses and deeper posterior regions. However, those deposition efficiencies are likely to be better for the pulsating airflow if the same integral airflow rate was used in the pulsating case compared to the steady case. Future studies should take this into account.

Moreover, the influence of one-way versus two-way coupling is studied. For the simulations with a steady inhalation of $7.5 \mathrm{~L} / \mathrm{min}$ and a particle diameter of $10 \mu \mathrm{m}$, the simulations with one-way and two-way coupling between two phases result in deposition efficiencies of $18.3 \%$ and $21.2 \%$, respectively. The higher DE is attributable to the higher gas velocity in case of two-way coupling since the drag forces causes a flow acceleration, leading to deeper penetration into the nasal cavity and thus, to a higher DE. Furthermore, the simple and the dynamic SGS using twoway coupling are compared for the steady inhalation with a particle diameter of $10 \mu \mathrm{m}$. The simulated values of DE with the simple and the dynamic Smagorinsky SGS are $21.2 \%$ and $24.8 \%$, respectively. The evaluation of these results is difficult due to a lack of experimental data for the deposition efficiency. However, due to the transitional laminar-turbulent character of the flow and its small length scales, it is likely that the dynamic Smagorinsky model leads to better results.

\section{Conclusions}

A study of the airflow and the dispersion and deposition of mono-disperse particles in a realistic model of a patient is performed using a large eddy simulation with dynamic sub-grid scale model and Lagrangian particle tracking. Both steady and pulsating airflows at the inlet are studied for different particle sizes. The results depict that the injection of larger particles leads to a higher deposition efficiency due to higher inertial impaction. Furthermore, it is observed that the DE is lower if one-way coupling between the gas phase and the particles is used compared to two-way coupling, where the influence of the particle motion on the gas is considered. Similarly, the simple Smagorinsky SGS model predicts a lower DE in comparison to the dynamic Smagorinsky SGS model.

The steady inhalation leads to particle deposition in the nasal valve, nasopharynx and parts of the septum, where the flow experiences a change in flow direction. For the pulsating airflow, a better ventilation of the meatuses and the olfactory regions is achieved compared with the steady inhalation. Even though the total DE is the same for the steady and the pulsating airflow, the perspective of the pulsating spray is better because of its improved ventilation of regions that are of particular interest for the medical treatment of critical diseases such as CRS. The deposition efficiency for the pulsating flow is likely to be enhanced if not the maximum value of the pulse is equal to the steady airflow velocity but the integral value, which would increase the current initial value of the pulsating flow by about a factor of 1.56 compared to the present value. Thus, the pulsating aerosol delivery is a potential drug delivery method for the CRS patients. Future studies concern the parametric dependence of the pulsation frequency and its amplitude on the DE of the drug delivery during pulsating drug delivery. 


\section{Acknowledgements}

The authors thank Dr. H. Mentzel from PARI Respiratory Equipment, Inc. for intense discussions about the pulsating spray initial conditions. The current study is part of a project supported by the German Research Foundation (DFG) through a research fellowship of the HGS MathComp. Furthermore, high-performance computing time at the bwForCluster MLS\&WISO Production which is funded by the state of Baden-Württemberg through bwHPC and DFG through grant INST 35/1134-1 FUGG.

\section{References}

[1] Tu, J., Inthavong, K., Ahmadi, G., 2013, "Computational Fluid and Particle Dynamics in the Human Respiratory System". Springer.

[2] Kimbell, J., Shroeter, D., Asgharian, B., Wong, B. A., Segal, R. A., Dickens, C. J., Southall, J. P. and Millerk F. J. 2004 Respiratory Drug Delivery, 9, pp. 233-238.

[3] Suman, J. D, 2013 Drug Delivery and Translational Research, 3, pp. 4-15.

[4] Shi, H., Kleinsteuer, C., Zhang, Z., 2007 Journal of Aerosol Science, 38, pp. 398-419.

[5] Fokkens, W. J., Lund, V. J., Mullol, J. Bachert, C., Alobid, I., Baroody, F., Cohen, N.,Cervin, A., Douglas, R.,Cevaert, P., Georgalas, C., Goossens, H., Harvey, R., Hellings, P., Hopkins, C.,Jones, N., Joos, G., Kalogjera, L., Kern, B., Kowalski, M., Price, D., Riechelmann, H., Schlosser, R., Senior, B., Thomas, M., Toskala, E., Voegels, R., Deyun, W., Wormald, P. J., 2012 Rhinology, Suppl. 23, pp. 1-299.

[6] Hastan, D., Fokkens, W. J., Bachert, C., Newson, R. B., Bislimovska, A., Bousquet, P. J., Brozek, G.,Dahlen, S. E., Forsberg, B., Gunnbjornsdottir, M.,Kasper, L., Krämer, U., Kowalski, M. L., Lange, B., Lundbäack, B., Salagean, E.,Todo-Bom, A.,Tomassen, P., Toskala, E.,van Drunen, C. M., Bousquet, J., Zuberbier, T., Jarvis, D., Burney, P., 2011 Allergy, 66, pp. 1216-1223.

[7] Blackwell, D. L., Lucas, J. W., and Clarke, T. C., 2014 National Center of Health Statistics, Vital Health Stat, 10(260).

[8] Iro, H., Mayr, S., Wällisch, C., Wigand, M. E., 2004 Rhinology, 42, pp. 200-206.

[9] Stammberger, H., Posawetz, G., 1990 European Archives of Oto-Rhino-Laryngology, 247, pp. 63-76.

[10] Baumann, I., Blumenstock, G., Praetorius, M., Sittel, C., Piccirillo, J. F., Plinkert, P. K., 2006 HNO, 54, pp. 544549.

[11] Möller, W., Schuschnig, U., Bartenstein, P., Meyer, G., Häussinger, K., Schmid, O., Becker, S., 2014 Journal of Aerosol Medicine and Pulmonary Drug Delivery, 27, pp. 255-263.

[12] Hilton, C., Wiedmann, T., Martin, M. S., Humphrey, B., Schleiffarth, R., Rimell, F., 2008 American Journal of Rhinology, 22, pp. 395-398.

[13] Proetz, A. W., 1951 Annals of Otology, Rhinology and Laryngology, 60, pp. 439-467.

[14] Elad, D., Liebenthal, R., Wenig, B. L., Einav, S., 1993 Med. Biol. Eng. Comput., 31, pp. 585-592.

[15] Swift, D. L., Storng, J. C., 1996 Journal of Aerosol Science, 27, pp. 1125-1996.

[16] Hornung, D. E., Leopold, D. A., Youngentob, S. L., Gagne, G. M., Thomas, F. D, 1987 Archives of Otolaryngology Head and Neck Surgery, 113, pp. 169-172.

[17] Hahn, I., Schere, P. W., Mozell, M. M., 1993 Annals of Otology, Rhinology and Laryngology, 75, pp. $2273-2287$.

[18] Kim, S. K., Chung, S. K., 2004 Measurement Science and Technology, 14, pp. 1090-1096.

[19] Doorly, D. J., Taylor, D. J., Schroter, R. C., 2008 Respiratory Physiology \& Neurobiology, 163, pp. 100-110.

[20] Keyhani, K., Scherer, P. W., Mozell, M. M., 1995 Journal of Biomedical Engineering, 117, pp. 429-441.

[21] Subramaniam, R. P., Richardson, R. B., Morgan, K. T., Kimbell, J. S., 1998 Inhalation Toxicology, 10, pp. 91-120.

[22] Liu, Y., Matida, E. A., Gu, J., Johnson, M. R., 2007 Journal of Aerosol Science, 38, pp. 683-700.

[23] Inthavong, K., Ge., Q., Se., C. M. K., Yang, W., Tu, J. Y., 2011 Journal of Aerosol Science, 42, pp. 100-113.

[24] Ghahramani, E., Abouali, O., Emdad, H., Ahmadi, G., 2014 Journal of Aerosol Science, 67, pp. 188-206.

[25] Moghadas, H., Abouali, O., Faramarzi, A., Ahmadi, G., 2011 Respiratory Physiology \& Neurobiology, 177, pp. 9-18.

[26] Zhu, J. H., Lim, K. M., Thong, K. T. M., Wang, D. Y., Lee, H. P., 2014 Respiratory Physiology \& Neurobiology, 194, pp. 29-36.

[27] Frank-Ito, D. O., Wofford, M., Schroeter, J. D., Kimbell, J. S., 2015 Journal of Aerosol Medicine and Pulmonary Drug Delivery, 28, pp. 1-11.

[28] Cui, X. G., 2012, "CFD Study of the Flow Field and Particle Dispersion and Deposition in the Upper Human Respiratory System". PhD Dissertation, Heidelberg University, Heidelberg, Germany.

[29] Germano, M., Piomelli, U., Moin, P., Cabot, W. H., 1991 Phys. Fluids, 3(7), pp. 1760-1765.

[30] Schiller, L., Naumann, A. Z., 1933 VDI Zeitschrift, 77, pp. 318-320.

[31] Pope, S.B., Turbulent Flows, first ed., Chambridge University Press, 2000.

[32] Edwards, D. A., Hanes, J., Caponetti, G., Hrkach, J., Ben-Jebria, A., Eskew, M. L., Mintzes, J., Deaver, D., Lotan, N. and Langer, R., 1997 Science, 276, pp. 1868-1872.

[33] Xi, J., Longest, P. W., 2009 Aerosol Science and Technology, 43, pp. 808-827.

[34] Shi, H., Kleinstreuer, C., Zhang, Z., 2006 Journal of Aerosol Science, 38, pp. 398-419.

[35] Farnoud, A., Cui, X. G., Baumann, I., Gutheil, E., May 22-27, 2016, 9th International Conference on Multiphase Flow, Florence, Italy.

[36] Chung, S. K., and Kim, S. K, 2008 Respiratory Physiology \& Neurobiology, 163, pp. 111-120. 